\title{
Psychometric Features of the Persian Version of the Fatigue Impact Scale in Iranian Stroke Patients
}

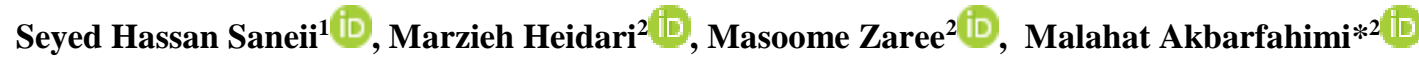

1. Dept. of Basic Sciences of Rehabilitation, School of Rehabilitation Sciences, Iran University of Medical Sciences, Tehran, Iran

2. Dept. of Occupational Therapy, School of Rehabilitation Sciences, Iran University of Medical Sciences, Tehran, Iran

\begin{tabular}{|c|}
\hline Article Info \\
\hline doi $10.30699 / j a m b s .28 .127 .111$ \\
\hline $\begin{array}{c}\text { Received: 2019/12/11; } \\
\text { Accepted: 2020/01/25; } \\
\text { Published Online: 01 Mar 2020; }\end{array}$ \\
\hline $\begin{array}{l}\text { Use your device to scan and read the } \\
\text { article online }\end{array}$ \\
\hline 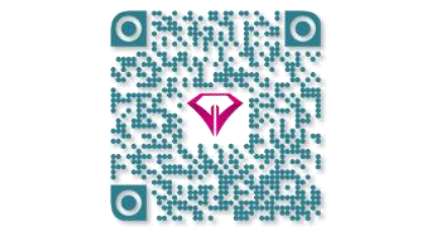 \\
\hline
\end{tabular}

Corresponding Information: Malahat Akbarfahimi, Dept. of Occupational Therapy, School of Rehabilitation Sciences, Iran University of Medical Sciences, Tehran, Iran

E-Mail: Akbarfahimi.m@iums.ac.ir

\section{ABSTRACT}

Background \& Objective: Post-stroke fatigue is a troublesome symptom and a common complaint of stroke patients. The purpose of this study was to investigate the psychometric features of the Persian version of the FIS (FIS-P) administered to Iranian stroke patients.

Materials \& Methods: In this cross-sectional methodological study, the psychometric properties of the Persian version of the FIS (FIS-P) were examined. The participants were 280 Iranian people (140 healthy adults; 140 stroke patients) who were assessed using the FIS-P, fatigue severity scale (FSS), and SF-36 questionnaire. The content, convergent, and discriminant validity of the FIS-P were evaluated using CVR and CVI, Pearson correlation, and independent-sampling T-tests, respectively. Furthermore, interrater reliability and test-retest reliability were assessed via the intra-class correlation coefficient and SEM. The internal consistency reliability was evaluated using Cronbach's $\alpha$.

Results: The results of the CVR for items ranged from 0.6-1, and the CVI of the FIS$\mathrm{P}$ was 0.85 . The Pearson correlation measure revealed strong and inverse correlations between FIS-P and FSS and a low to moderate relationship between all subscales of the SF-36 and FIS-P in terms of convergent validity. T-test results showed the discriminant validity of the FIS-P in differentiating between stroke patients and healthy participants. The ICC coefficients for test-retest and inter-rater reliability for the overall scale were 0.991 and 0.984 , respectively. Cronbach's $\alpha$ was 895 .

Conclusion: The strong psychometric properties of the FIS-P indicated its applicability in assessing the impact of fatigue on stroke victim's daily activities and the effectiveness of therapeutic and rehabilitation interventions.

Keywords: Fatigue, Stroke, Reliability, Validity

\section{Introduction}

Strokes are among the most common neurological diseases (1). Based on the lesion location, stroke survivors can suffer from various physical and cognitive impairments and emotional disturbances that can limit their functional abilities $(2,3)$. Post-stroke fatigue (PSF) is recognized as a common symptom, affecting $23-75 \%$ of stroke survivors (4). PSF is a debilitating condition that can cause lowered selfesteem, sleep disorders, anxiety, cognitive disorders, and mood disturbances, which adversely affects the victim's quality of life, social participation, return to work, and mortality (4-6). Hence, early PSF diagnosis and interventions could increase stroke survivors' quality of life and improve their physical and cognitive abilities (7). Although various tools for assessing fatigue are available, PSF lacks an approved goldstandard outcome (8). Therefore, the assessment of fatigue is challenging in two regards. The first challenge is related to the lack of a standardized scale for research purposes, and the second has to do with the intervention aims of the rehabilitation team and the absence of a tool for evaluating the impact of fatigue on stroke survivors' daily lives (9).

There are a lot of fatigue assessment tools available, such as the fatigue severity scale (FSS) (10), fatigue impact scale (FIS) (11), fatigue assessment scale (FAS) (12), and fatigue scale for motor and cognitive functions (FSMC) (13). Most of them were originally designed to assess fatigue related to multiple sclerosis (MS). However, some of them are commonly used (with accurate results) for other groups of patients in other research fields, such in clinical settings $(14,15)$.

The FIS is a commonly used self-report scale that was developed by Fisk et al. in 1994 to evaluate the impact of fatigue on the everyday activities of MS patients $(\mathbf{1 1}, 16)$. The FIS has been translated and validated in 30 languages and has been applied to 
evaluate fatigue among patients suffering from strokes, brain concussions, poliomyelitis, chronic fatigue syndrome, lupus, and hepatitis (17). The FIS is highly suitable for people who tend to control their fatigue and who try to identify the dimensions of their lives that are affected by fatigue (18). The scale contains 40 items that assess the fatigue-related limitations in the patients' performance in cognitive (10 questions), physical (10 questions), and social (20 questions) activity domains. Studies have been carried out in different languages, such as American English (19), Hungarian (20), Turkish (21), and French (22). The FIS has also been translated into Persian and validated for MS patients (23).

The purpose of the present study is to determine whether the Persian version of the FIS (FIS-P) can be applied as a valid scale for measuring the effects of therapeutic interventions on fatigue control among stroke survivors. In this study, we aimed to assess the face, content, discriminant, and convergent validity, as well as the ceiling and flooring effects and reliability (test-retest, inter-rater, and internal sensitivity) of the FIS-P when administered to Persian-speaking stroke patients in Iran.

\section{Materials and Methods}

\section{Design}

In this cross-sectional methodological research carried out Mar-Sep 2015 and the psychometric features of the FIS (FIS-P) were investigated, with Persian-speaking stroke patients in Iran comprising the study population. The ethical protocol of this study followed the Declaration of Helsinki. The process was approved by the institutional review board of Iran University of Medical Sciences. All participants signed a written informed consent prior to enrollment.

\section{Participants}

The participants included 140 stroke patients who were referred to Tehran Occupational Therapy Clinics from 2015-2016, as well as 140 healthy adults who were matched with the stroke patients for age, sex, and marital status using the convenience sampling method. To be included in the study, stroke victims must have had their diagnosis confirmed by an expert neurologist and MRI report, must have had their first stroke during the study period, six months to five years must have elapsed since their stroke, and they needed to have an FSS score of greater than 4 (6).

Inclusion criteria for all participants were an age range of 45-70 years, the ability to read and write, adequate cooperation, an MMSE score of greater than 21, and Persian had to be their native language.

Exclusion criteria were a history of substance abuse; comorbidity with psychiatric, orthopedic, and neurological disorders (any lesions and anomalies in the central nervous system), sleep deprivation, chronic fatigue syndrome and other similar diseases. All participants denied using anti-fatigue medication. Data provided on incomplete questionnaires and scales were excluded.

Content validity was assessed by 20 occupational experts, each of whom had more than seven years of experience in stroke rehabilitation and at least five papers published in this field.

\section{Setting}

For all stages of this study, the assessment sessions for both groups were implemented in a quiet room in occupational therapy clinics between 8:00 a.m. and 1:00 p.m. The purpose of the research was clarified at the beginning of the session.

\section{Procedures}

\section{Translation}

The FIS was translated into Persian (24) and its psychometric version was examined in patients with multiple sclerosis (23). In this study, we used the FIS$\mathrm{P}$ and examined the psychometric features in the stroke population.

\section{Face validity}

In order to determine the face validity of the scale, 20 patients with stroke stated the relevancy, suitability, clarity, and simplicity of each item of FIS-P Scale (24). In the end, an occupational therapist asked them to explain their perceptions of each item. Our approach for face validity was interpretability of the items (25).

\section{Content validity}

To control the content validity of the scale, 20 expert occupational therapists identified the essentiality and relevancy of each item of FIS-P (26).

\section{Convergent validity}

140 patients with stroke took part to determine the convergent validity of the FIS-P scale and fulfilled the FIS-P, FSS and SF, 36 in random order.

\section{Discriminant validity}

In order to define the discriminant validity of FIS-P between the normal population and stroke, 140 healthy adults and 140 patients with stroke were assessed using FIS-P.

\section{Interrater Reliability}

During two sessions in one day, two assessors, blinded to the aim of the study, asked 20 patients with dominant hemiplegia due to stroke to complete the FIS$\mathrm{P}$. The assessors read the items, explained the items if the participants needed clarification and filled in the blanks.

Test-retest reliability: A total of 40 patients with stroke fulfilled the FIS-P two times with a one-week interval. 


\section{Measures}

We used the Persian version of the scales/ questionnaire in this study, and the process of the translation was based on International Quality of Life Assessment (IQOLA).

\section{Fatigue Impact Scale (FIS)}

The FIS-P was translated by Heidari et al. (24); permission from Mapi Research Trust was granted by MAPI (http://www.mapi-trust.org.). This scale includes 40 items on three subscales. The cognitive impact of fatigue subscale contains 10 items and focuses on the concentration, memory, thinking, and organization of thoughts. The physical impact of fatigue subsection includes 10 items and reflects motivations, endeavors, tolerance, and coordination. The social impact of fatigue subscale includes 20 items and describes the effects of fatigue on isolation, emotion, stress, and communication. Possible scores ranged from 0 (no problem) to 4 (extreme problem). For the entire scale, possible scores ranged from 0-160 points, with higher scores indicating more problems. The intra-class correlation (ICC) values for inter-rater reliability on the physical subscale, cognitive subscale, social subscale, and total score were 0.89, 0.86, 0.95, and 0.98 , respectively; the test-retest reliability values were $0.86,0.78,0.92$, and 0.93 , respectively. The Cronbach's alpha of the FIS-P was 0.95 , which indicates the high reliability of the FIS-P (23). The questionnaire takes 10-20 minutes to complete and five minutes to score.

\section{Fatigue Severity Scale (FSS)}

This self-reported scale is comprised of nine items. The participants were to state their feelings over the past two weeks for each item, and scores could range from 1-7 points. High disagreement with an item yields a low score, while a strong agreement with an item yields a high score. The internal consistency of the items on the Persian version of the FSS was 0.96 based on Cronbach's alpha coefficient. The correlation of each item with another item was 0.4. The ICC coefficient was 0.93 (27).

\section{Short-form health survey (SF-36)}

The SF-36 questionnaire consists of two main subgroups of items (physical and mental health), with each of these subgroups including four domains. The physical subgroup includes the domains of physical function (10 items), role limitation (four items), body pain (seven items), and general health (four items). The mental health subgroup includes the domains of social function (two items), role emotion (three items), vitality (four items), and mental health (five items). Scores on the SF-36 scale can vary between 0 and 100 , with higher scores representing a higher level of healthrelated to one's quality of life (28). The Persian version of the SF-36 was used in the present study. The internal consistency analysis of the Persian version of the SF36 ranged from $0.77-0.90$ (except for in the vitality domain). The correlation coefficient is greater than the recommended value of 0.4 (the coefficients ranged from 58.8-0.95). This survey is currently the most widely used instrument for measuring the quality of life in the world (29).

\section{Statistical Analysis}

The statistical analysis was accomplished using SPSS 16 (IBM Inc., Chicago, IL., USA). Content validity rate (CVR) and content validity index (CVI) were used as measures of content validity (26). The results of the Kolmogorov-Smirnov test determined the normal distribution of the FIS-P, FSS, and SF-36. Therefore, the Pearson correlation and independentsamples T-test were applied to examine convergent and discriminant validity, respectively. Cronbach's alpha coefficients were used to assess internal consistency, and ICC was applied to determine inter-rater and testretest reliability. Based on ICC correlation coefficients, reliability was interpreted as follows: $<0.4=$ weak, 0.4 $0.7=$ fine, $>0.7=$ great $(30)$. Cronbach's alpha scores were defined as follows: for $>0.9=$ excellent, $0.7-0.9=$ good, 0.6-0.7 = acceptable, 0.5-0.6 = weak, and $<0.5=$ non-acceptable. (30). Furthermore, the standard error of measurement (SEM) was calculated to estimate measurement precision. SEM and MDC were calculated using the following equations: $M D C=$ $\operatorname{SEM} \sqrt{ }(2 \pm 1.96)$ and $\operatorname{SEM}=(\operatorname{SD} \sqrt{ }(1-I C C)(31,32)$. A P-value of less than 0.05 was considered significant.

\section{Results}

The two groups (140 healthy adults and 140 stroke patients) were matched in terms of their age, sex, and marital status $(P<0.05)$. The mean age \pm standard deviation of the stroke patients was $58.85 \pm 7.88$, and that of the healthy adults was $58.16 \pm 10.51(P=0.553)$. Of the stroke patients, 91 had right hemiplegia. The time since the stroke in the stroke group was 20.27 \pm 15.82 months (Table 1).

\section{Face validity}

The relevance, suitability, clarity, and simplicity of all questions were acceptable.

\section{Content validity}

The CVR of the items ranged from 0.6-1, which is acceptable according to Lawshe's method (26) with 20 experts which is 0.42 . The average CVI of the scale (SCVI/Ave) was 0.85 , and the universal agreement of the scale CVI (S-CVI/UA) was 0.48 . Of the 40 items on the scale, 19 gained the universal agreement of experts.

\section{Floor and ceiling effects}

The percentage of floor and ceiling effects for the FIS-P subscales and the total score was $2.1 \%$. Therefore, it can be concluded that the FIS-P does not 
have a ceiling or floor effect for the stroke patients in this study.

\section{Convergent validity}

Table 2 presents the Pearson correlation results, which revealed significant negative correlations between the FIS-P (subscales and total score) and all SF-36 subscales, with the only exception found for the cognitive subscale of the FIS-P and the emotional domain of the SF-36. The FIS-P and FSS scales had a significant positive correlation.

\section{Discriminant validity}

Table 3 contains the results regarding the significant differences between stroke patients and healthy adults regarding their scores on all three subscales (as well as their total scores) of the FIS-P based on the independent-sampling T-test $(P<0.05)$.

\section{Test-retest reliability}

The ICC coefficient results revealed large reliability correlation coefficients for the physical (0.961), cognitive (0.987), social (0.987), and total (0.991) scores of the FIS-P (Table 4).

\section{Inter-rater reliability}

The ICC coefficients revealed strong reliability correlation coefficients for the physical (0.911), cognitive (0.987), social (0.987), and overall (0.984) FIS-P scores (Table 4). Cronbach's alpha coefficient was used to assess the internal consistency of the FIS test. The Cronbach's $\alpha$ of the total scale was 0.953 , indicating the high reliability of the results of the FIS$\mathrm{P}$ in stroke patients in this study.

Table 1. The demographic characteristics of stroke patients $(n=140)$ and healthy adults $(n=140)$

\begin{tabular}{|c|c|c|c|c|c|c|}
\hline \multirow[t]{2}{*}{ Variable } & \multirow[t]{2}{*}{ Groups } & \multicolumn{2}{|c|}{$\begin{array}{c}\text { Stroke Patients } \\
\quad(n=140)\end{array}$} & \multicolumn{2}{|c|}{$\begin{array}{l}\text { Healthy adults } \\
\qquad(\mathrm{n}=140)\end{array}$} & \multirow[t]{2}{*}{$\boldsymbol{P}$} \\
\hline & & $\mathrm{N}$ & Percent & $\mathrm{N}$ & Percent & \\
\hline \multirow{2}{*}{ Gender } & Male & 71 & 50.7 & 77 & 55 & \multirow{2}{*}{0.27} \\
\hline & Female & 69 & 49.3 & 63 & 45 & \\
\hline \multirow{2}{*}{ Marital status } & Single & 101 & 72.1 & 100 & 71.4 & \multirow{2}{*}{0.5} \\
\hline & Married & 39 & 27.9 & 40 & 28.6 & \\
\hline \multirow{2}{*}{ Educational status(class) } & $\geq 12$ & 124 & 88.6 & 108 & 77.1 & \multirow{2}{*}{0.017} \\
\hline & $<12$ & 16 & 11.4 & 32 & 24.9 & \\
\hline \multirow{3}{*}{ Hemiplegia side } & Right & 91 & 65 & - & - & \multirow{2}{*}{$<0.001$} \\
\hline & Left & 49 & 35 & - & - & \\
\hline & & Mean & SD & Mean & SD & $P$ \\
\hline Age & & 58.85 & 7.88 & 58.16 & 10.51 & 0.553 \\
\hline Time since stroke & & 20.27 & 15.82 & - & - & \\
\hline
\end{tabular}

Table 2. Pearson Correlations of the Persian Version of the Fatigue Impact scale (FIS-P) with the Quality of Life Scale (SF-36) and Fatigue Severity Scale (FSS)

\begin{tabular}{|c|c|c|c|c|}
\hline \multirow{3}{*}{ Subscales/Scale } & \multicolumn{3}{|c|}{ FIS-P Subscales } & \multirow{2}{*}{$\begin{array}{l}\text { FIS-P } \\
\text { Total }\end{array}$} \\
\hline & Cognitive & Physical & Social & \\
\hline & $\mathrm{r}$ & c & & $\mathrm{r}$ \\
\hline \multicolumn{5}{|l|}{ SF36 } \\
\hline Physical Function & $-0.525 * *$ & $-0.480 * *$ & $-0.549 * *$ & $-0.546 * *$ \\
\hline Role physical & $-0.249 * *$ & $0.175 * *_{-}$ & $-0.228 * *$ & $-0.229 * *$ \\
\hline Role Emotional & NS & $-0.174 * *$ & $0.188 * *_{-}$ & $-0.184 * *$ \\
\hline vitality & $-0.465 * *$ & $-0.455 * *$ & $-0.517 * *$ & $-0.508 * *$ \\
\hline Mental Health & $0.332 * *_{-}$ & $-0.342 * *$ & $-0.384 * *$ & $-0.375^{* *}$ \\
\hline Social Function & $-0.400 * *$ & $-0.412 * *$ & $-0.423 * *$ & $-0.431 * *$ \\
\hline Physical pain & $-0.420 * *$ & $-0.452 * *$ & $-0.466 * *$ & $-0.469 * *$ \\
\hline General Health & $-0.452 * *$ & $0.463 * *_{-}$ & $0.501 * *_{-}$ & $0.498 * *_{-}$ \\
\hline FSS & $0.697 * *$ & $0.731 * *$ & $0.771 * *$ & $0.772 * *$ \\
\hline
\end{tabular}


Table 3. Comparison of the FIS-P scores of healthy adults $(n=140)$ and stroke patients $(n=140)$

\begin{tabular}{lcccccc} 
FIS-P & \multicolumn{2}{c}{$\begin{array}{c}\text { Stroke Patients } \\
(\mathrm{n}=140)\end{array}$} & \multicolumn{2}{c}{$\begin{array}{c}\text { Healthy adults } \\
(\mathrm{n}=140)\end{array}$} & \multicolumn{2}{c}{$\begin{array}{c}\text { independent-Sampling T } \\
\text { test }\end{array}$} \\
& $\mathrm{M}$ & $\mathrm{SD}$ & $\mathrm{M}$ & $\mathrm{SD}$ & $\mathrm{t}$ & $P$ \\
Total score & 73.22 & 34.19 & 47.96 & 33.39 & 256.6 & $<0.001$ \\
Physical & 22.75 & 8.95 & 14.81 & 9.64 & 7.14 & $<0.001$ \\
Cognitive & 14.87 & 8.97 & 10.13 & 8.64 & 4.50 & $<0.001$ \\
Social & 35.59 & 17.63 & 23.01 & 16.91 & 6.09 & $<0.001$ \\
\hline
\end{tabular}

Data was shown as Mean and SD(Standard Deviation, t: independent-Sampling T test,

P:P-value, FIS: Fatigue Impact Scale, Physical: Physical Domain subscale of FIS, Cognitive: Domain subscale of FIS, Social: Domain subscale of FIS

Table 4. Descriptive statistics and reliability statistics for the FIS-P

\begin{tabular}{|c|c|c|c|c|c|c|c|c|c|}
\hline \multirow{2}{*}{ FIS-P } & \multirow{2}{*}{$\begin{array}{l}\text { Cronbach' } \\
\text { s Alpha }\end{array}$} & \multicolumn{3}{|c|}{ Inter rater } & \multicolumn{5}{|c|}{ Test-retest } \\
\hline & & ICC & $95 \% \mathrm{CI}$ & $P$ & ICC & $95 \% \mathrm{CI}$ & $P$ & SEM & MDC \\
\hline Total & 0.895 & 0.984 & $\begin{array}{c}0.848- \\
0.848\end{array}$ & 0.001 & 0.991 & $\begin{array}{c}0.983- \\
0.995\end{array}$ & $<0.001$ & 2.99 & 8.26 \\
\hline Physical & 0.87 & 0.911 & $\begin{array}{c}0.142- \\
0.991\end{array}$ & 0.019 & 0.961 & $\begin{array}{c}0.979- \\
0.926\end{array}$ & $<0.001$ & 1.40 & 3.87 \\
\hline Cognitive & 0.90 & 0.987 & $\begin{array}{c}0.879- \\
0.999\end{array}$ & $<0.001$ & 0.987 & $\begin{array}{c}0.993- \\
0.976\end{array}$ & $<0.001$ & 1.00 & 2.76 \\
\hline Social & 0.95 & 0.987 & $\begin{array}{c}0.876- \\
0.999\end{array}$ & $<0.001$ & 0.987 & $\begin{array}{c}0.976- \\
0.993\end{array}$ & $<0.001$ & 1.91 & 5.27 \\
\hline
\end{tabular}

CI: Confidence interval, ICC: Intra-class Correlation Coefficient, P:P-value, FIS-P: Persian Version of Fatigue Impact Scale, Physical: Physical Domain subscale of FIS, Cognitive: Domain subscale of FIS, Social: Domain subscale of FIS, SEM: Standard Error of Measurement, MDC: Minimum Detectable Change.

\section{Discussion}

Most stroke survivors suffer from post-stroke fatigue, and the early detection (and subsequent interventions) of the impacts of fatigue on a patient's life can play a crucial role in reducing its negative effects. Thus, having a valid and reliable fatigue assessment tool is essential. This study investigated the validity and reliability of the Persian version of the fatigue impact scale (FIS-P) in stroke patients in Iran. According to our results, the FIS-P is a widely used scale in research and clinical settings that has acceptable validity and reliability for assessing fatigue among stroke survivors.

To assess the validity of the FIS-P, we examined face validity, content validity, convergent validity, divergent validity, and floor and ceiling effects.

Face validity: Based on the opinions of the stroke patients, all items on the FIS-P are suitable, easy to understand, and unambiguous. This result is consistent with previous reports from patients with MS $(23,24)$.

Content validity: The FIS was originally designed to be completed by MS patients. The experts examined each item and confirmed all items were essential and relevant for assessing the impact of fatigue in stroke patients. The high S-CVI/UA score (0.48) indicated that all experts who participated in this study reached a universal agreement for 19 items. Therefore, the FIS-P can be used to assess the impact of fatigue in stroke victims' lives.

Another property of the FIS-P was lack of noticeable floor and ceiling effects in all subscales and total scores for stroke patients.

Convergent validity: $\mathrm{We}$ assessed convergent validity by examining the relationship between FIS-P, FSS, and SF-36 scores. The FSS and FIS-P scores were strongly positively correlated. This means that both assessed a common concept. This result corroborates the findings of other researchers $(\mathbf{1 9}, 23)$. However, the association was slightly weaker in these other studies, perhaps because of the different study populations (MS patients instead of stroke patients). Furthermore, FIS$\mathrm{P}$ and SF-36 were used to evaluate the convergent validity of the FIS-P. Inverse and low to moderate relationships were found between all subscales and total scores of the FIS-P and SF-36, with the one exception being the correlation between the cognitive domain subscale of the FIS-P and the emotional role subscale of the SF-36 questionnaire. This inverse correlation was considered an exception because fatigue reduces the quality of life of stroke patients, as 
has been reported in many studies (5). However, the low to moderate relationships between these two tools suggests that they are somewhat structurally different, as have been reported previously (19).

Discriminative validity: The mean FIS-P score of the stroke group was significantly greater than that in the healthy group in all domains of fatigue. This means that the FIS-P can differentiate stroke patients from healthy adults in terms of their fatigue. In agreement with Heidari et al.'s study, the FIS-P has an acceptable discriminant validity (23).

To assess the reliability of the FIS-P, the test-retest reliability, inter-rater reliability, and internal consistency were examined.

Test-retest reliability: Test-retest reliability was assessed both in terms of the absolute and related values of reliability using SEM and ICC, respectively. The subscale scores and total FIS-P score have a reasonable ICC level, which is consistent with other studies that used other versions of the FIS, such as those carried out by Heidari et al., Mathiowetz et al., Armutlu et al., and Losonczi et al. (19,21-23). The ICC value obtained in the present work signifies that the FIS-P has satisfactory test-retest reliability. Also, no significant changes were found between the test and retest means of subscales and total scores. The low SEM values resulted in low MDC values for the FIS-P.

Inter-rater reliability: The calculation of inter-rater reliability is not common for self-report tools completed by stroke victims due to the severity of the disease and because most stroke patients suffer from hemiplegia. Furthermore, some stroke survivors have tremors or visual impairments, making it difficult for them to read or write. These stroke survivors need a second party to read the questionnaire items to them and write their responses. Because of this, inter-rater reliability needs to be evaluated. The ICC results for the FIS-P showed strong agreement between two raters. This implication of this reliability is in a clinical setting.

Internal consistency: The Cronbach's coefficient $\alpha$ values were high for the total FIS-P score (0.895) and for its subscales (0.911-0.987). This result is consistent with other studies carried out in different languages such as Hungarian (Cronbach's $\alpha$ of 0.98) (20), Turkish (Cronbach's $\alpha$ of 0.97 and 0.91 for the total score and subscales, respectively) (21), and French (Cronbach's $\alpha$ of 0.80 ) (22). Therefore, it can be concluded that the tool is consistent with the original tool and can be used in stroke patients.

\section{Limitations}

Like many other investigations, the current study had limitations. The use of the convenience sampling method and the lack of a previous Persian version of a similar instrument to assess convergent validity are noteworthy limitations of this study.

\section{Conclusion:}

Given the strong psychometric features of FIS in comparison to other tools, the Persian version of the FIS (FIS-P) is recommended as a suitable tool for assessing fatigue. This tool may also be applied to study the impact of treatment and rehabilitation interventions on fatigue in Persian-speaking stroke patients. The use of the FIS-P questionnaire in local research centers is therefore recommended.

\section{Acknowledgment}

This study is a report of an independent research project in Iran University of Medical Sciences entitled "Validity and Reliability of the Persian Version of Fatigue Impact Scale in Patients with Stroke "encoded 93.04.32.25325. The authors would like to thank all participants.

\section{Conflict of Interest}

Authors declared no conflict of interest.

\section{References}

1. Virani SS, Alonso A, Benjamin EJ, et al. Heart disease and stroke statistics-2020 Update: A report from the American Heart Association. Circulation. 2020;141(9):e139-e596.

2. Ramsey LE, Siegel JS, Lang CE, StrubeM, Shulman GL, Corbetta M. Behavioural clusters and predictors of performance during recovery from stroke. Nat Hum Behav. 2017;1 [DOI:10.1038/s41562-0160038]

3. Akbari S, Lyden PD, Kamali M, Fahimi MA. Correlations among impairment, daily activities and thinking operations after stroke. NeuroRehabilitation. 2013;33(1):153-60. [DOI:10.3233/NRE-130940]

4. Kjeverud A, Ostlie K, Schanke AK, Gay C, Thoresen M, Lerdal A. Trajectories of fatigue among stroke patients from the acute phase to 18 months post-injury: A latent class analysis. PLoS One. 2020;15(4):e0231709. [DOI:10.1371/journal.pone.0231709]

5. Cumming $\mathrm{TB}$, Yeo AB, Marquez $\mathrm{J}$, et al. Investigating post-stroke fatigue: An individual participant data meta-analysis. J Psychosom Res. 2018;113:107-12. [DOI:10.1016/j.jpsychores.2018.08.006]

6. Naess H, Lunde L, Brogger J, Waje-Andreassen U. Fatigue among stroke patients on long-term followup. The Bergen Stroke Study. J Neurol Sci. 2012;312(1-2):138-41. [DOI:10.1016/j.jns.2011.08.002] 
7. Duncan F, Wu S, Mead GE. Frequency and natural history of fatigue after stroke: a systematic review of longitudinal studies. J Psychosom Res. 2012;73(1):18-27.

[DOI:10.1016/j.jpsychores.2012.04.001]

8. Thomas K, Hjalmarsson C, Mullis R, Mant J. Conceptualising post-stroke fatigue: a crosssectional survey of UK-based physiotherapists and occupational therapists. BMJ Open. 2019;9(12):e033066. [DOI:10.1136/bmjopen-2019033066]

9. Mead G, Lynch J, Greig C, Young A, Lewis S, Sharpe M. Evaluation of fatigue scales in stroke patients. Stroke. 2007;38(7):2090-5. [DOI:10.1161/STROKEAHA.106.478941]

10. Krupp LB, LaRocca NG, Muir-Nash J, Steinberg AD. The fatigue severityscale. Application to patients with multiple sclerosis and systemic lupus erythematosus. Arch Neurol. 1989;46(10):1121-3. [DOI:10.1001/archneur.1989.00520460115022]

11. Fisk JD, Pontefract A, Ritvo PG, Archibald CJ, Murray TJ. The impact of fatigue on patients with multiple sclerosis. Canadian J Neurol Sci . 1994;21(1):9-14. [DOI:10.1017/S0317167100048691]

12. Michielsen HJ, De Vries J, Van Heck GL. Psychometric qualities of a brief self-rated fatigue measure: The Fatigue Assessment Scale. J Psychosom Res. 2003;54(4):345-52. [DOI:10.1016/S0022-3999(02)00392-6]

13. Penner IK, Raselli C, Stocklin M, Opwis K, Kappos $\mathrm{L}$, Calabrese P. The fatigue scale for motor and cognitive functions (FSMC): validation of a new instrument to assess multiple sclerosis-related fatigue. Mult Scler. 2009;15(12):1509-17 [DOI:10.1177/1352458509348519]

14. Elf M, Eriksson G, Johansson S, von Koch L, Ytterberg C. Self-reported fatigue and associated factors six years after stroke. PLoS One. 2016;11(8):e0161942.

[DOI:10.1371/journal.pone.0161942]

15. Parks NE, Eskes GA, Gubitz GJ, Reidy Y, Christian C, Phillips SJ. Fatigue impactscale demonstrates greater fatigue in younger stroke survivors Canadian J Neurol Sci. 2012;39(5):619-25. [DOI:10.1017/S0317167100015353]

16. Fisk JD, Ritvo PG, Ross L, Haase DA, Marrie TJ, Schlech WF. Measuring thefunctional impact of fatigue: initial validation of the fatigue impact scale. Clin Infect Dis. 1994;18 Suppl 1:S79-83. [DOI:10.1093/clinids/18.Supplement 1.S79]

17. Frith J, Newton J. Fatigue Impact Scale. Occup Med. 2010;60(2):159. [DOI:10.1093/occmed/kqp180]
18. Fisk JD, Doble SE. Construction and validation of a fatigue impact scale for daily administration (DFIS). Quality of life research . Qual life Res. 2002;11(3):263-72. [DOI:10.1023/A:1015295106602]

19. . Mathiowetz V. Test-retest reliability and convergent validity of the fatigue impact scale for persons with multiple sclerosis. Am J Occup Ther. 2003;57(4):389-95. [DOI:10.5014/ajot.57.4.389]

20. Losonczi E, Bencsik K, Rajda C, Lencses G, Torok $\mathrm{M}$, VecseiL. Validation of the fatigue impact scale in Hungarian patients with multiple sclerosis. Qual Life Res. 2011;20(2):301-6. [DOI:10.1007/s11136010-9749-7]

21. . Armutlu K, Keser I, Korkmaz N, et al. Psychometric study of Turkish version of fatigue impact scale in multiple sclerosis patients. J Neurol Sci. 2007;255(1-2):64-8. [DOI:10.1016/j.jns.2007.01.073]

22. Debouverie M, Pittion-Vouyovitch S, Louis S, Guillemin F. Validity of a french version of the fatigue impact scale in multiple sclerosis. Mult Scler. 2007;13(8):1026-32. [DOI:10.1177/1352458507077942]

23. Heidari M, Akbarfahimi M, Salehi M, Nabavi SM. Validity and reliability of the persian-version of fatigue impact scale in multiple sclerosis patients in Iran. koomesh. 2014;15(3):295-301.

24. Heidari M, Akbarfahimi M, Salehi M. Survey validity of translation for Persian and cultural adaptation of Fatigue Impact Scale (FIS) questionnaire in multiple sclerosis patients in Iran. J Modern Rehabil. 2012;6(320-29).

25. Terwee CB, Bot SD, de Boer MR, et al. Quality criteria were proposed for measurement properties of health status questionnaires. J Clin Epidemiol. 2007;60(1):34-42. [DOI:10.1016/j.jclinepi.2006.03.012]

26. Lawshe $\mathrm{CH}$. A quantitative approach to content validity. Personnel Psychol. 1975;28:563-75. [DOI:10.1111/j.1744-6570.1975.tb01393.x]

27. Azimian M, Farahani AS, Dadkhah A, Fallahpour M, Karimlu M. Fatigue severity scale: the psychometric properties of the persian-version in patients with multiple sclerosis. Res J Biol Sci 2009;4:974-7.

28. Ware JE, Sherbourne CD. The MOS 36-item shortform health survey (SF-36). I. Conceptual framework and item selection. Med Care. 1992;30(6):473-83. [DOI:10.1097/00005650199206000-00002] 
29. Montazeri A, Goshtasebi A, Vahdaninia M, Gandek B. The short form health survey (SF-36): translation and validation study of the Iranian version. Qual Life Res. 2005;14(3):875-82. [DOI:10.1007/s11136004-1014-5]

30. Feliss J. Design and analysis of clinical experiments. New York: John Wiley \& Sons; 1986.

31. Liu WY, Hou YJ, Liao HF, Lin YH, Chen YY, Wong AM. A Preliminary study of the development, validity, and reliability of a caregiver questionnaire for the health-related quality of life in children with cerebral palsy. Chang Gung Med J. 2010;33(6):64658 .

\section{How to Cite This Article:}

Saneii S H, Heidari M, Zaree M, Akbarfahimi M. Psychometric Features of the Persian Version of the Fatigue Impact Scale in Iranian Stroke Patients. J Adv Med Biomed Res. 2020; 28 (127) :111-118

\section{Download citation:}

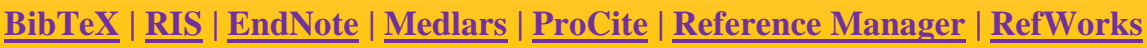

\section{Send citation to:}

Q Mendeley 2 Zotero ;)RefWorks $\underline{\text { RefWorks }}$
32. Mehdizadeh M, Martinez-Martin P, Habibi SA, et al. Reliability and validity of fall efficacy scaleinternational in people with parkinson's disease during on- and off-drug phases. Parkinson's disease. 2019;2019:6505232. [DOI:10.1155/2019/6505232] 